\title{
Existence of Lipschitz continuous Lyapunov functions strict outside the strong chain recurrent set
}

\author{
OLGA BERNARDI ${ }^{1} \quad$ ANNA FLORIO $^{2}$ \\ ${ }^{1}$ Dipartimento di Matematica "Tullio Levi-Civita", Università di Padova, \\ Via Trieste, 63 - 35121 Padova, Italy \\ ${ }^{2}$ Laboratoire de Mathématiques d'Avignon, Avignon Université, \\ 84018 Avignon, France
}

\begin{abstract}
The aim of this paper is to study in detail the relations between strong chain recurrence for flows and Lyapunov functions. For a continuous flow on a compact metric space, uniformly Lipschitz continuous on the compact subsets of the time, we first make explicit a Lipschitz continuous Lyapunov function strict -that is strictly decreasing- outside the strong chain recurrent set of the flow. This construction extends to flows some recent advances of Fathi and Pageault in the case of homeomorphisms; moreover, it improves Conley's result about the existence of a continuous Lyapunov function strictly decreasing outside the chain recurrent set of a continuous flow. We then present two consequences of this theorem. From one hand, we characterize the strong chain recurrent set in terms of Lipschitz continuous Lyapunov functions. From the other hand, in the case of a flow induced by a vector field, we establish a sufficient condition for the existence of a $C^{1,1}$ strict Lyapunov function and we also discuss various examples. Moreover, for general continuos flows, we show that the strong chain recurrent set has only one strong chain transitive component if and only if the only Lipschitz continuous Lyapunov functions are the constants. Finally, we provide a necessary and sufficient condition to guarantee that the strong chain recurrent set and the chain recurrent one coincide.
\end{abstract}

\section{Introduction}

Let $\phi=\left\{\phi_{t}\right\}_{t \in \mathbb{R}}$ be a continuous flow on a compact metric space $(X, d)$. In the paper 9$][$ Page 96], Robert Easton introduced the notion of strong chain recurrence.

DEFINITION. (Strong chain recurrence)

(i) Given $x, y \in X, \varepsilon>0$ and $T>0$, a strong $(\varepsilon, T)$-chain from $x$ to $y$ is a finite sequence $\left(x_{i}, t_{i}\right)_{i=1, \ldots, n} \subset X \times \mathbb{R}$ such that $t_{i} \geq T$ for all $i, x_{1}=x$ and setting $x_{n+1}=y$ we have

$$
\sum_{i=1}^{n} d\left(\phi_{t_{i}}\left(x_{i}\right), x_{i+1}\right)<\varepsilon .
$$

(ii) A point $x \in X$ is said to be strong chain recurrent if for all $\varepsilon>0$ and $T>0$ there exists a strong $(\varepsilon, T)$-chain from $x$ to $x$. The set of strong chain recurrent points is denoted by $\operatorname{SCR}(\phi)$.

(iii) The points $x, y \in \mathcal{S C R}(\phi)$ belong to the same strong chain transitive component of $\mathcal{S C R}(\phi)$ if for any $\varepsilon>0$ and $T>0$ there exist a strong $(\varepsilon, T)$-chain from $x$ to $y$ and a strong $(\varepsilon, T)$-chain from $y$ to $x$. 
This notion sharpens the one of chain recurrence, in which it is only required

$$
d\left(\phi_{t_{i}}\left(x_{i}\right), x_{i+1}\right)<\varepsilon
$$

for all $i$. Chain recurrent sets are both easily seen to be closed and invariant, see e.g. 2][Pages 12-13, 71 and 109-110], [8][Theorem 3.3B], 20][Page 248] and [6][Proposition 3.1]. Furthermore, strong chain recurrent set (strictly) contains the set of non-wandering points and it is (strictly) contained in the chain recurrent set, that is $\mathcal{N} \mathcal{W}(\phi) \subseteq \mathcal{S C R}(\phi) \subseteq \mathcal{C R}(\phi)$, see for example [20][Theorem 2.4] and [8][Theorem 3.3B].

The study of the intimate relations between chain recurrent sets and Lyapunov functions comes from the celebrated paper [7] by Charles Conley and has had recent important advances by Albert Fathi and Pierre Pageault in [12] and [16]. Before recalling these results, we need to premise the notions of Lyapunov function, neutral set and first integral.

DEFINITION. (Lyapunov function, neutral set and first integral)

A function $f: X \rightarrow \mathbb{R}$ is a Lyapunov function for $\phi$ if $f \circ \phi_{t} \leq f$ for every $t \geq 0$. The neutral set of a Lyapunov function $f$ is

$$
\mathcal{N}(f):=\left\{x \in X: \exists t>0 \text { such that } f\left(\phi_{t}(x)\right)=f(x)\right\} .
$$

A function $f: X \rightarrow \mathbb{R}$ is a first integral for $\phi$ if $f \circ \phi_{t}=f$ for every $t \in \mathbb{R}$.

In particular, $f$ is a first integral if and only if $\mathcal{N}(f)=X$. We refer to Lemma 1.5 in [1] for a characterization of Lyapunov functions and first integrals in the case of a flow induced by a locally Lipschitz continuous vector field.

In the seminal paper [7], Conley described the structure of the chain recurrent set in terms of attractors and their "complementary repellers" and -as an outcome- he proved the theorem below. Some authors refer to this result as the "fundamental theorem of dynamical systems", see e.g. [15] and [13] for an instructive treatment of the matter.

THEOREM. (Conley, 1978)

Let $\phi: X \times \mathbb{R} \rightarrow X$ be a continuous flow on a compact metric space. Then there exists a continuous Lyapunov function $u: X \rightarrow \mathbb{R}$ for $\phi$ such that

(i) $\mathcal{N}(u)=\mathcal{C R}(\phi)$.

(ii) If $x, y \in \mathcal{C R}(\phi)$, then $u(x)=u(y)$ if and only if $x$ and $y$ belong to the same chain transitive component of $\mathcal{C} \mathcal{R}(\phi)$.

(iii) $u(\mathcal{C R}(\phi))$ is a compact nowhere dense subset of $\mathbb{R}$.

Conley called $u$ complete Lyapunov function for $\phi$, see Definition 5.2 of Section 4. In particular, point $(i)$ of the previous theorem is an either/or statement about what can happen: parts of the space are either chain recurrent or "gradient-like". In the same year -see [9][Proposition 3]Easton connected the notion of strong chain recurrence to the property for the corresponding Lipschitz first integrals to be constants. Easton's contribution represented the first step towards the study of the relations between strong chain recurrence and Lyapunov functions.

For a homeomorphism $g$ on a compact metric space, Fathi and Pageault in [12] and [16] presented a new variational point of view to face the study of recurrent sets and Lyapunov functions. Their techniques are very different from Conley's original ones and are inspired by Fathi's work in weak KAM theory, see [10]. Indeed, thanks to the arbitrariness of the parameter $\varepsilon>0$ involved in both the definitions of recurrence, Fathi and Pageault equivalently described recurrent points as minima of appropriate functionals defined on the space of finite sequences of points. In particular, bearing in mind formulae (1) and (2), for strong chain recurrent points the 
functional is the sum of the amplitudes of the jumps; for chain recurrent points, the functional is the maximum of the amplitudes of the jumps. For a discrete dynamical system, if $u$ is a Lyapunov function for $g$, that is $u \circ g \leq u$, the neutral set of $u$ is

$$
\mathcal{N}(u):=\{x \in X: u(g(x))=u(x)\} .
$$

We remind two of their fundamental results. On one hand, they provided a new proof of the above Conley's theorem in the framework of discrete-time dynamical systems. On the other hand, they proved the next

THEOREM. (Fathi \& Pageault, 2015).

Let $g: X \rightarrow X$ be a homeomorphism on a compact metric space. Then

(i) There exists a Lipschitz continuous Lyapunov function u for $g$ such that $\mathcal{N}(u)=\mathcal{S C R}(g)$.

(ii) $\mathcal{S C R}(g)=\bigcap \mathcal{N}(f)$, where the intersection is taken over all Lipschitz continuous Lyapunov functions $f$ for $g$.

(iii) $\operatorname{SCR}(g)$ has a unique strong chain transitive component if and only if the only Lipschitz continuous Lyapunov functions for $g$ are the constants.

We refer respectively to Theorem 4.4, Corollary 4.5 and Theorem 4.8 in [16 for the proofs of the points $(i)$, (ii) and (iii) above. More recently, an adaptation of Fathi and Pageault's techniques has led to the proof of point $(i i)$ of the previous theorem also for a flow which is Lipschitz continuous for every $t \geq 0$, uniformly for $t$ on compact subsets of $[0,+\infty)$, see [1][Theorem 2.2]. However, in [1] it is not constructed a single Lyapunov function whose neutral set coincides with the strong chain recurrent set. We finally underline that, more recently, Fathi and Pageault in [?] give a criterion for the approximation of a Lyapunov function by a smooth one and also show that it is possible to obtain a smooth version of Conley's fundamental theorem for flows.

This paper intends to examine in depth the relations between strong chain recurrence and Lyapunov functions in the case of flows. First, we prove this improvement of point $(i)$ of Conley's original result:

THEOREM 1. Let $\phi: X \times \mathbb{R} \rightarrow X$ be a continuous flow on a compact metric space $(X, d)$, uniformly Lipschitz continuous on the compact subsets of $[0,+\infty)$. Then there exists a Lipschitz continuous Lyapunov function $u: X \rightarrow \mathbb{R}$ for $\phi$ such that

$$
\mathcal{N}(u)=\mathcal{S C R}(\phi) .
$$

The proof of this theorem combines together variational and dynamical methods. In particular, we start by constructing -by accurately adapting Fathi and Pageault's techniques- a function which strictly decreases along the flow out of a closed set containing $\mathcal{S C R}(\phi)$ and definitively (that is, for $t \geq T$ ) in time. We refer to Proposition 3.1 for the precise statement. Thereafter, by using some ideas coming from the original proof of Conley's theorem, we modify this function in order to obtain the desired Lyapunov function, that is strictly decreasing outside $\operatorname{SCR}(\phi)$ for any $t>0$. See Lemma 4.1, Lemma 4.2 and the consequent Theorem 4.1 .

We proceed by discussing the main consequences of the above theorem. From one hand, we give an alternative -i.e. constructive- proof of Theorem 2.2 in [1]:

COROLlARY 1. Let $\phi: X \times \mathbb{R} \rightarrow X$ be a continuous flow on a compact metric space $(X, d)$, uniformly Lipschitz continuous on the compact subsets of $[0,+\infty)$. Then

$$
\operatorname{SCR}(\phi)=\bigcap \mathcal{N}(f)
$$


where $f$ is any Lipschitz continuous Lyapunov function for $\phi$.

From the other hand, we discuss a sufficient condition in order to establish the existence of a $C^{1,1}$ strict Lyapunov function for a flow induced by a vector field:

COROLLARY 2. Let $(M, g)$ be a $C^{\infty}$ closed connected Riemannian manifold. Let $V: M \rightarrow T M$ be a $C^{k}$ vector field, $k \geq 2$, inducing the flow $\phi$. If $\mathcal{S C R}(\phi)=\mathcal{C} \mathcal{R}(\phi)$ then there exists a $\mathcal{C}^{1,1}$ Lyapunov function $u: M \rightarrow \mathbb{R}$ for $\phi$ such that $\mathcal{N}(u)=\operatorname{SCR}(\phi)$.

See respectively Corollary 4.1 and Corollary 4.2 . In particular, the proof of the second corollary uses existence and regularity results for sub-solutions of the so-called Mañé Hamiltonians. We then pass to discuss some cases -Examples 4.1 and 4.2 where the hypothesis $\mathcal{S C R}(\phi)=\mathcal{C R}(\phi)$ of this corollary is not satisfied and any sub-solutions of weak KAM theory provide first integrals, while the Lyapunov function previously constructed is not a first integral.

We also remark that the converse implication of this corollary does not in general hold true. To be thorough, in Definition 5.2 we remind the milder notion of pseudo-complete Lyapunov function and in Proposition 5.3 we prove a necessary and sufficient condition for the strong chain recurrent and the chain recurrent sets to be equal:

PROPOSITION. Let $\phi: X \times \mathbb{R} \rightarrow X$ be a continuous flow on a compact metric space $(X, d)$. Then, $\operatorname{SCR}(\phi)=\mathcal{C R}(\phi)$ if and only if there exists a pseudo-complete Lyapunov function $u: X \rightarrow$ $\mathbb{R}$ for $\phi$ such that $u(\mathcal{S C R}(\phi))$ is totally disconnected.

We finally analyze the relations between Lipschitz continuous Lyapunov functions and the strong chain transitive components of $\mathcal{S C R}(\phi)$. As a final outcome -see Theorem 5.1- we show that point (iii) of Fathi and Pageault's theorem still holds in the case of a continuous flow:

THEOREM 2. Let $\phi: X \times \mathbb{R} \rightarrow X$ be a continuous flow on a compact metric space $(X, d)$. $\operatorname{SCR}(\phi)$ has a unique strong chain transitive component if and only if the only Lipschitz continuous Lyapunov functions for $\phi$ are the constants.

Acknowledgements. O. Bernardi has been supported by the project CPDA149421/14 of the University of Padova. O. Bernardi and A. Florio acknowledge the support of G.N.F.M. The authors also thank the anonymous referee for the careful reading of the manuscript and his/her many insightful comments and suggestions.

\section{The function $L_{T}$ : definition and properties}

Let $\phi: X \times \mathbb{R} \rightarrow X$ be a continuous flow on a compact metric space $(X, d)$. The next definitions are the continuous-time versions of the ones introduced by Fathi and Pageault for homeomorphisms, see [16][Chapter 2, Section 3] and [12][Section 2.1]. We also underline that their settings have recently been extended by Ethan Akin and Jim Wiseman in [3] both to relations and to uniform spaces.

Let $x, y \in X$. For any $T>0$, we indicate by $C_{T}(x, y)$ the set of chains $C=\left(x_{i}, t_{i}\right)_{i=1, \ldots, n} \subset$ $X \times \mathbb{R}$ from $x_{1}=x$ to $x_{n+1}=y$ such that $t_{i} \geq T$ for all $i$. The cost of going from $x$ to $y$ through a chain $C \in C_{T}(x, y)$ is given by

$$
l_{T}(C):=\sum_{i=1}^{n} d\left(\phi_{t_{i}}\left(x_{i}\right), x_{i+1}\right)
$$


Moreover, for any $T>0$, we define the non-negative function

$$
\begin{aligned}
L_{T} & : X \times X \rightarrow[0,+\infty) \\
L_{T}(x, y) & :=\inf \left\{l_{T}(C): C \in C_{T}(x, y)\right\} .
\end{aligned}
$$

In the next proposition, we summarize some useful facts about the function $L_{T}$. We refer to [16][Proposition 3.1] and [12][Proposition 2.1] for analogous results in the case of a homeomorphism.

Proposition 2.1. Let $\phi: X \times \mathbb{R} \rightarrow X$ be a continuous flow on a compact metric space $(X, d)$. For any fixed $T>0$, the following properties hold:

(i) For any $x, y, z \in X$,

$$
L_{T}(x, y) \leq L_{T}(x, z)+L_{T}(z, y)
$$

(ii) For any $x \in X$,

$$
L_{T}\left(x, \phi_{t}(x)\right)=0 \quad \forall t \geq T
$$

(iii) For any $x, y, z \in X$,

$$
\left|L_{T}(x, y)-L_{T}(x, z)\right| \leq d(y, z) .
$$

As a consequence, for any fixed $x \in X$, the function $z \mapsto L_{T}(x, z)$ is 1-Lipschitz continuous.

(iv) For any fixed $x \in X$, the function $z \mapsto L_{T}(z, x)$ is upper semicontinuous.

Proof. For points $(i),(i i)$ and $(i i i)$ we refer to Lemma 2.4 in [1].

(iv) By definition of $L_{T}$, for any $z \in X$ and $\varepsilon>0$, from the definition of infimum, there exists a chain $C_{\varepsilon} \in C_{T}(z, x)$ such that

$$
l_{T}\left(C_{\varepsilon}\right)<L_{T}(z, x)+\frac{\varepsilon}{2} .
$$

Let $t_{1}=t_{1}(z) \geq T$ be the first time of the chain $C_{\varepsilon}$. Since $\phi_{t_{1}}$ is a homeomorphism, for any $\varepsilon>0$ there exists $\delta=\delta(\varepsilon)>0$ so that

$$
d(z, y)<\delta \quad \Rightarrow \quad d\left(\phi_{t_{1}}(z), \phi_{t_{1}}(y)\right)<\frac{\varepsilon}{2} .
$$

Let us consider the chain $\tilde{C}_{\varepsilon} \in C_{T}(y, x)$ obtained from $C_{\varepsilon}$ by substituting the first point with $y$. Hence, if $d(z, y)<\delta$, we have that

$$
L_{T}(y, x) \leq l_{T}\left(\tilde{C}_{\varepsilon}\right) \leq d\left(\phi_{t_{1}}(z), \phi_{t_{1}}(y)\right)+l_{T}\left(C_{\varepsilon}\right)<\varepsilon+L_{T}(z, x) .
$$

For any $T>0$, we define the subset $\mathcal{A}_{T}$ of $X$ as

$$
\mathcal{A}_{T}:=\left\{x \in X: L_{T}(x, x)=0\right\} .
$$

Since $C_{T}(x, y)$ is contained in $C_{T^{\prime}}(x, y)$ when $T \geq T^{\prime}$, the function $T \mapsto L_{T}(x, y)$ is monotonically increasing for any pair $(x, y) \in X \times X$ and $\mathcal{A}_{T} \subseteq \mathcal{A}_{T^{\prime}}$. We notice that

$$
\operatorname{SCR}(\phi)=\bigcap_{T>0} \mathcal{A}_{T}
$$

The next proposition characterizes the points of the strong chain recurrent set in terms of the functions $L_{T}$.

Proposition 2.2. Let $\phi: X \times \mathbb{R} \rightarrow X$ be a continuous flow on a compact metric space $(X, d)$.

(i) If $x \in \mathcal{S C R}(\phi)$, then $L_{T}\left(\phi_{t}(x), x\right)=L_{T}\left(x, \phi_{t}(x)\right)=0$ for any $T>0$ and $t \in \mathbb{R}$. 
(ii) If for any $T>0$ there exists a time $t=t(T) \in \mathbb{R}$ such that $L_{T}\left(\phi_{t}(x), x\right)=L_{T}\left(x, \phi_{t}(x)\right)=0$, then $x \in \mathcal{S C R}(\phi)$.

In particular, if $x \in \mathcal{S C R}(\phi)$ then $x$ and $\phi_{t}(x)$ belong to the same strong chain transitive component of $\mathcal{S C R}(\phi)$ for any $t \in \mathbb{R}$.

Proof. (i) Let $x \in \mathcal{S C R}(\phi), T>0$ and $t \in \mathbb{R}$ be fixed and take $N=N(T, t) \in \mathbb{N}$ such that $t \leq N T$. Since $x \in \mathcal{S C R}(\phi), L_{(N+1) T}(x, x)=0$. This means that for any $\varepsilon>0$ there exists a chain $C_{\varepsilon}=\left(x_{i}, t_{i}\right)_{i=1, \ldots, n} \in C_{(N+1) T}(x, x)$ such that

$$
l_{(N+1) T}\left(C_{\varepsilon}\right) \leq \varepsilon .
$$

Let consider the following chain $\tilde{C}_{\varepsilon} \in C_{T}\left(\phi_{t}(x), x\right)$ obtained from $C_{\varepsilon}$ :

$$
\tilde{C}_{\varepsilon}=\left(\left(\phi_{t}(x), t_{1}-t\right),\left(x_{2}, t_{2}\right), \ldots,\left(x_{n}, t_{n}\right)\right) .
$$

Then it clearly holds that

$$
0 \leq L_{T}\left(\phi_{t}(x), x\right) \leq l_{T}\left(\tilde{C}_{\varepsilon}\right)=l_{(N+1) T}\left(C_{\varepsilon}\right) \leq \varepsilon
$$

From the arbitrariness of $\varepsilon>0$, we deduce that $L_{T}\left(\phi_{t}(x), x\right)=0$. Since the above argument can be repeated for any fixed $x \in \mathcal{S C R}(\phi), T>0$ and $t \in \mathbb{R}$, we conclude that if $x \in \mathcal{S C R}(\phi)$ then $L_{T}\left(\phi_{t}(x), x\right)=0$ for any $T>0$ and $t \in \mathbb{R}$.

For a fixed $x \in \mathcal{S C R}(\phi), T>0$ and $t \in \mathbb{R}$, we now prove the other equality. If $t \geq T$, the fact that $L_{T}\left(x, \phi_{t}(x)\right)=0$ corresponds exactly to property $(i i)$ of Proposition 2.1. Consequently, let $t<T$ and choose $N=N(T, t) \in \mathbb{N}$ such that $T<N|t|$. Moreover, observe that by the invariance of the strong chain recurrent set, $\phi_{t}(x) \in \mathcal{S C R}(\phi)$. This means that, for any $\varepsilon>0$ there exists a chain $C_{\varepsilon}=\left(x_{i}, t_{i}\right)_{i=1, \ldots, n} \in C_{(N+1)|t|}\left(\phi_{t}(x), \phi_{t}(x)\right)$ such that

$$
l_{(N+1)|t|}\left(C_{\varepsilon}\right) \leq \varepsilon .
$$

Let us consider the following chain $\tilde{C}_{\varepsilon} \in C_{T}\left(x, \phi_{t}(x)\right)$ obtained from $C_{\varepsilon}$ :

$$
\tilde{C}_{\varepsilon}=\left(\left(x, t_{1}+t\right),\left(x_{2}, t_{2}\right), \ldots,\left(x_{n}, t_{n}\right)\right),
$$

so that

$$
0 \leq L_{T}\left(x, \phi_{t}(x)\right) \leq l_{T}\left(\tilde{C}_{\varepsilon}\right)=l_{(N+1)|t|}\left(C_{\varepsilon}\right) \leq \varepsilon .
$$

From the arbitrariness of $\varepsilon>0$, we conclude that $L_{T}\left(x, \phi_{t}(x)\right)=0$. Since the above argument holds for any fixed $x \in \mathcal{S C R}(\phi), T>0$ and $t<T$, we conclude that if $x \in \operatorname{SCR}(\phi)$ then $L_{T}\left(x, \phi_{t}(x)\right)=0$ for any $T>0$ and $t \in \mathbb{R}$.

(ii) Conversely, for a point $x \in X$, let assume that for any $T>0$ there exists a time $t=t(T) \in \mathbb{R}$ such that

$$
L_{T}\left(\phi_{t}(x), x\right)=L_{T}\left(x, \phi_{t}(x)\right)=0
$$

Thanks to property $(i)$ of Proposition 2.1, we have that

$$
0 \leq L_{T}(x, x) \leq L_{T}\left(x, \phi_{t}(x)\right)+L_{T}\left(\phi_{t}(x), x\right)=0 .
$$

From the arbitrariness of $T>0$, we deduce that $x \in \mathcal{S C R}(\phi)$. 


\section{Construction of Lipschitz Lyapunov functions definitively strict}

Through the whole Sections 3 and 4 , we assume that $\phi: X \times \mathbb{R} \rightarrow X$ is a continuous flow on a compact metric space, uniformly Lipschitz continuous on every compact subset of $[0,+\infty)$. This means that for any $T>0$ there exists $M_{T}>0$ such that

$$
d\left(\phi_{t}(x), \phi_{t}(y)\right) \leq M_{T} d(x, y) \quad \forall t \in[0, T] .
$$

The above Lipschitz regularity assumption is surely satisfied by the flow of a Lipschitz continuous vector field on a compact manifold. Moreover, we precise that we can make a measurable choice of the function $s \mapsto M_{s}$, for instance

$$
M_{s}:=\max \left(\sup _{t \in[0, s], x \neq y} \frac{d\left(\phi_{t}(x), \phi_{t}(y)\right)}{d(x, y)}, 1\right) .
$$

Let $\mathcal{K}:=\left\{x_{1}, \ldots, x_{j}, \ldots\right\}$ be a countable dense subset of $X$; such a set exists since $X$ is a compact metric space. For any $T>0$, we define the function

$$
\begin{aligned}
u_{T} & : X \rightarrow \mathbb{R} \\
u_{T}(x) & :=\sum_{j \in \mathbb{N}} \frac{1}{2^{j}} L_{T}\left(x_{j}, x\right) .
\end{aligned}
$$

We underline that this technique is the same of Fathi and Pageault, see [12][Proposition 2.3] and [16][Chapter 2, Proposition 4.1]. Clearly, the function $u_{T}$ is bounded. Indeed, for any $x \in X$, it holds that

$$
\left|u_{T}(x)\right| \leq \sum_{j \in \mathbb{N}} \frac{1}{2^{j}} L_{T}\left(x_{j}, x\right) \leq \sum_{j \in \mathbb{N}} \frac{1}{2^{j}} d\left(\phi_{T}\left(x_{j}\right), x\right) \leq 2 \operatorname{diam}(X)
$$

where $\operatorname{diam}(X)=\max \{d(x, y): x, y \in X\}<+\infty$. In the next proposition we summarize the properties of $u_{T}$.

Proposition 3.1. Let $\phi: X \times \mathbb{R} \rightarrow X$ be a continuous flow on a compact metric space $(X, d)$, uniformly Lipschitz continuous on compact subsets of $[0,+\infty)$. For any fixed $T>0$, the following properties hold:

(i) $u_{T}$ is 2-Lipschitz continuous.

(ii) $u_{T}$ is definitively a Lyapunov function for $\phi$, that is

$$
u_{T}\left(\phi_{t}(x)\right) \leq u_{T}(x) \quad \text { for any } x \in X \text { and } t \geq T .
$$

(iii) $u_{T}$ is definitively strict outside $\mathcal{A}_{\frac{T}{2}}$, that is

$$
u_{T}\left(\phi_{t}(x)\right)<u_{T}(x) \quad \text { for any } x \in X \backslash \mathcal{A}_{\frac{T}{2}} \text { and } t \geq T \text {. }
$$

Proof. (i) Thanks to property (iii) of Proposition 2.1, for any $x, y \in X$ it holds that

$$
\left|u_{T}(y)-u_{T}(x)\right| \leq \sum_{j \in \mathbb{N}} \frac{1}{2^{j}}\left|L_{T}\left(x_{j}, y\right)-L_{T}\left(x_{j}, x\right)\right| \leq 2 d(x, y)
$$

(ii) By point $(i)$ of Proposition 2.1, for any $x, y \in X$ we have that

$$
u_{T}(y)-u_{T}(x)=\sum_{j \in \mathbb{N}} \frac{1}{2^{j}}\left(L_{T}\left(x_{j}, y\right)-L_{T}\left(x_{j}, x\right)\right) \leq 2 L_{T}(x, y)
$$


For $y=\phi_{t}(x)$ and $t \geq T$, we obtain

$$
u_{T}\left(\phi_{t}(x)\right)-u_{T}(x) \leq 2 L_{T}\left(x, \phi_{t}(x)\right)=0
$$

where -in the last equality- we use property (ii) of Proposition 2.1.

(iii) Arguing by contradiction, let us suppose that $u_{T}$ is not definitively strict outside $\mathcal{A}_{\frac{T}{2}}$. This means that there exist $z \in X \backslash \mathcal{A}_{\frac{T}{2}}$ and $s \geq T$ such that

$$
u_{T}\left(\phi_{s}(z)\right)-u_{T}(z)=\sum_{j \in \mathbb{N}} \frac{1}{2^{j}}\left(L_{T}\left(x_{j}, \phi_{s}(z)\right)-L_{T}\left(x_{j}, z\right)\right)=0 .
$$

Since from properties $(i)$ and $(i i)$ of Proposition 2.1, it holds

$$
L_{T}\left(x_{j}, \phi_{t}(x)\right)-L_{T}\left(x_{j}, x\right) \leq 0 \quad \forall x \in X, j \in \mathbb{N} \text { and } t \geq T,
$$

hypothesis $(9)$ equals to

$$
L_{T}\left(x_{j}, \phi_{s}(z)\right)-L_{T}\left(x_{j}, z\right)=0 \quad \forall j \in \mathbb{N} .
$$

Let $m \in \mathbb{N}$ be fixed. In the sequel we prove that there exists $x_{j(m)} \in \mathcal{K}$ such that

$$
L_{T}\left(x_{j(m)}, \phi_{s}(z)\right)<\frac{1}{m} .
$$

Notice that, since $\mathcal{K}$ is a dense sequence in $X$, it is always possible to take $x_{j(m)} \in \mathcal{K}$ such that $d\left(x_{j(m)}, z\right)<\frac{1}{m M_{2 T}}$. By hypothesis 6 , this implies

$$
d\left(\phi_{t}\left(x_{j(m)}\right), \phi_{t}(z)\right)<\frac{1}{m} \quad \forall t \in[0,2 T] .
$$

We now explicitly exhibit a chain $C \in C_{T}\left(x_{j(m)}, \phi_{s}(z)\right)$ such that

$$
l_{T}(C)<\frac{1}{m} .
$$

From one hand, if $s \in[T, 2 T]$ then $C=\left(x_{j(m)}, s\right)$. Indeed, by [12), we have

$$
l_{T}(C)=d\left(\phi_{s}\left(x_{j(m)}\right), \phi_{s}(z)\right)<\frac{1}{m} .
$$

From the other hand, if $s>2 T$, let consider the chain $C \in C_{T}\left(x_{j(m)}, \phi_{s}(z)\right)$ defined as follows:

$$
C=\left(\left(x_{j(m)}, T\right),\left(\phi_{T}(z), s-T\right)\right) .
$$

By (12) again, it holds

$$
l_{T}(C)=d\left(\phi_{T}\left(x_{j(m)}\right), \phi_{T}(z)\right)+d\left(\phi_{s}(z), \phi_{s}(z)\right)<\frac{1}{m}+0=\frac{1}{m} .
$$

Since $L_{T}\left(x_{j(m)}, \phi_{s}(z)\right) \leq l_{T}(C)$ for any $C \in C_{T}\left(x_{j(m)}, \phi_{s}(z)\right)$, inequality (11) immediately follows. Consequently -see $(10)$ - we obtain:

$$
L_{T}\left(x_{j(m)}, z\right)<\frac{1}{m} .
$$

By using previous inequality, we are now going to conclude that $L_{\frac{T}{2}}(z, z)=0$. Given an arbitrary chain $C \in C_{T}\left(x_{j(m)}, z\right)$ from $x_{j(m)}$ to $z$ :

$$
C=\left(\left(x_{j(m)}, t_{1}\right),\left(y_{2}, t_{2}\right), \ldots,\left(y_{n}, t_{n}\right)\right),
$$


let us consider the chain $\tilde{C} \in C_{\frac{T}{2}}(z, z)$ obtained by $C$ and defined as follows:

$$
\tilde{C}=\left(\left(z, \frac{T}{2}\right),\left(\phi_{\frac{T}{2}}\left(x_{j(m)}\right), t_{1}-\frac{T}{2}\right),\left(y_{2}, t_{2}\right), \ldots,\left(y_{n}, t_{n}\right)\right) .
$$

By 12 , we have

$$
L_{\frac{T}{2}}(z, z) \leq l_{\frac{T}{2}}(\tilde{C})=d\left(\phi_{\frac{T}{2}}(z), \phi_{\frac{T}{2}}\left(x_{j(m)}\right)\right)+d\left(\phi_{t_{1}}\left(x_{j(m)}\right), y_{2}\right)+\cdots+d\left(\phi_{t_{n}}\left(y_{n}\right), z\right)<\frac{1}{m}+l_{T}(C) .
$$

Since $L_{T}\left(x_{j(m)}, z\right)<\frac{1}{m}$, by taking the infimum over all possible chains in $C_{T}\left(x_{j(m)}, z\right)$, we have

$$
L_{\frac{T}{2}}(z, z) \leq \frac{1}{m}+\frac{1}{m}=\frac{2}{m} .
$$

From the arbitrariness of $m \in \mathbb{N}$, we obtain $L_{\frac{T}{2}}(z, z)=0$. Since $z \in X \backslash \mathcal{A}_{\frac{T}{2}}$, previous equality gives the desired contradiction.

\section{Existence of Lipschitz Lyapunov functions with $\mathcal{S C R}(\phi)$ as neu- tral set}

For a continuous flow $\phi: X \times \mathbb{R} \rightarrow X$ satisfying hypothesis (6), this section is devoted to prove the existence of a Lipschitz continuous Lyapunov function which is strict outside $\operatorname{SCR}(\phi)$. The proof -see Theorem 4.1 - is preceded by two technical lemmas aiming to overcome the fact that the function (8) constructed in Proposition 3.1 is only definitively a strict Lyapunov function for $\phi$. We then proceed to discuss some consequences of Theorem 4.1 and to give various examples.

For any $T>0$, we start by defining

$$
\begin{aligned}
\tilde{u}_{T} & : X \rightarrow \mathbb{R} \\
\tilde{u}_{T}(x) & :=\max _{s \in[0, T]} u_{T}\left(\phi_{s}(x)\right)
\end{aligned}
$$

and

$$
\begin{aligned}
\bar{u}_{T} & : X \rightarrow \mathbb{R} \\
\bar{u}_{T}(x) & :=\frac{1}{M_{T}} \int_{0}^{+\infty} \frac{e^{-s}}{M_{s}} \tilde{u}_{T}\left(\phi_{s}(x)\right) d s .
\end{aligned}
$$

where the function $s \mapsto M_{s}$ has been defined in (7). We remark that the above definitions are inspired by the ones given by Conley in the proof of the so-called fundamental theorem of dynamical systems, see [7][Chapter II, Section 5, Page 33] and [Chapter II, Section 6, Page 39]. The main properties of functions (14) and (15) are presented in the next two lemmas.

Lemma 4.1. Let $\phi: X \times \mathbb{R} \rightarrow X$ be a continuous flow on a compact metric space $(X, d)$, uniformly Lipschitz continuous on compact subsets of $[0,+\infty)$. For any fixed $T>0$, the following properties hold:

(i) $\tilde{u}_{T}$ is $2 M_{T}$-Lipschitz continuous.

(ii) $\tilde{u}_{T}$ is a Lyapunov function for $\phi$.

(iii) $\tilde{u}_{T}$ is definitively strict outside $\mathcal{A}_{\frac{T}{2}}$, that is

$$
\tilde{u}_{T}\left(\phi_{t}(x)\right)<\tilde{u}_{T}(x) \quad \text { for any } x \in X \backslash \mathcal{A}_{\frac{T}{2}} \text { and } t \geq T \text {. }
$$


Proof. Thanks to property $(i)$ of Proposition 3.1 and assumption (6) on the flow, for any $x, y \in X$

$$
\begin{gathered}
\tilde{u}_{T}(y)-\tilde{u}_{T}(x)=\max _{s \in[0, T]} u_{T}\left(\phi_{s}(y)\right)-\max _{s \in[0, T]} u_{T}\left(\phi_{s}(x)\right) \leq \max _{s \in[0, T]}\left(u_{T}\left(\phi_{s}(y)\right)-u_{T}\left(\phi_{s}(x)\right)\right) \leq \\
\leq 2 \max _{s \in[0, T]} d\left(\phi_{s}(y), \phi_{s}(x)\right) \leq 2 M_{T} d(x, y) .
\end{gathered}
$$

We conclude by exchanging the role of $x$ and $y$.

(ii) By property (ii) of Proposition 3.1, we know that

$$
u_{T}\left(\phi_{\tau}(x)\right) \leq \max _{s \in[0, T]} u_{T}\left(\phi_{s}(x)\right) \quad \forall x \in X \text { and } \tau \geq 0 .
$$

By taking the maximum for $\tau \in[t, T+t]$, we obtain

$$
\max _{\tau \in[t, T+t]} u_{T}\left(\phi_{\tau}(x)\right) \leq \max _{s \in[0, T]} u_{T}\left(\phi_{s}(x)\right)=\tilde{u}_{T}(x)
$$

Since now

$$
\max _{\tau \in[t, T+t]} u_{T}\left(\phi_{\tau}(x)\right)=\max _{s \in[0, T]} u_{T}\left(\phi_{s+t}(x)\right)=\tilde{u}_{T}\left(\phi_{t}(x)\right),
$$

the thesis immediately follows.

(iii) By property (iii) of Proposition 3.1, for any $x \in X \backslash \mathcal{A}_{\frac{T}{2}}$ and $t \geq T$, we have

$$
\tilde{u}_{T}\left(\phi_{t}(x)\right)=\max _{s \in[0, T]} u_{T}\left(\phi_{s+t}(x)\right)<u_{T}(x) \leq \max _{s \in[0, T]} u_{T}\left(\phi_{s}(x)\right)=\tilde{u}_{T}(x) .
$$

Lemma 4.2. Let $\phi: X \times \mathbb{R} \rightarrow X$ be a continuous flow on a compact metric space $(X, d)$, uniformly Lipschitz continuous on compact subsets of $[0,+\infty)$. For any fixed $T>0$, the following properties hold:

(i) $\bar{u}_{T}$ is 2-Lipschitz continuous.

(ii) $\bar{u}_{T}$ is a Lyapunov function for $\phi$.

(iii) $\bar{u}_{T}$ is strict outside $\mathcal{A}_{\frac{T}{2}}$ for any $t>0$.

Proof. (i) By property ( $i$ ) of Lemma 4.1 and assumption (6) on the flow, we immediately obtain that for all $x, y \in X$

$\bar{u}_{T}(y)-\bar{u}_{T}(x)=\frac{1}{M_{T}} \int_{0}^{+\infty} \frac{e^{-s}}{M_{s}}\left(\tilde{u}_{T}\left(\phi_{s}(y)\right)-\tilde{u}_{T}\left(\phi_{s}(x)\right)\right) d s \leq 2 \int_{0}^{+\infty} \frac{e^{-s}}{M_{s}} d\left(\phi_{s}(y), \phi_{s}(x)\right) d s \leq 2 d(y, x)$.

We conclude by exchanging the role of $x$ and $y$.

(ii) The statement is a direct consequence of property (ii) of Lemma 4.1. Indeed, for any $x \in X$ and $t \geq 0$, it clearly holds

$$
\bar{u}_{T}\left(\phi_{t}(x)\right)-\bar{u}_{T}(x)=\frac{1}{M_{T}} \int_{0}^{+\infty} \frac{e^{-s}}{M_{s}}\left(\tilde{u}_{T}\left(\phi_{s+t}(x)\right)-\tilde{u}_{T}\left(\phi_{s}(x)\right)\right) d s \leq 0
$$

(iii) By definition,

$$
\bar{u}_{T}\left(\phi_{t}(x)\right)-\bar{u}_{T}(x)=\frac{1}{M_{T}} \int_{0}^{+\infty} \frac{e^{-s}}{M_{s}}\left(\tilde{u}_{T}\left(\phi_{s+t}(x)\right)-\tilde{u}_{T}\left(\phi_{s}(x)\right)\right) d s .
$$

Let us introduce

$$
\bar{s}:=\max \left\{s \in[0,+, \infty]: \tilde{u}_{T}\left(\phi_{s}(x)\right)=\tilde{u}_{T}(x)\right\}
$$


and notice that -by property (iii) of Lemma $4.1-\bar{s} \in[0, T)$. Consequently, for any $x \in X \backslash \mathcal{A}_{\frac{T}{2}}$ and $t>0$, we have

$$
\tilde{u}_{T}\left(\phi_{\bar{s}+t}(x)\right)<\tilde{u}_{T}(x)=\tilde{u}_{T}\left(\phi_{\bar{s}}(x)\right) .
$$

By the previous strict inequality, we deduce that

$$
\bar{u}_{T}\left(\phi_{t}(x)\right)-\bar{u}_{T}(x)<0 \quad \forall x \in x \in X \backslash \mathcal{A}_{\frac{T}{2}} \text { and } t>0 .
$$

We finally prove

Theorem 4.1. Let $\phi: X \times \mathbb{R} \rightarrow X$ be a continuous flow on a compact metric space $(X, d)$, uniformly Lipschitz continuous on compact subsets of $[0,+\infty)$. Then

$$
\begin{gathered}
u: X \rightarrow \mathbb{R} \\
x \mapsto u(x):=\sum_{n \in \mathbb{N}} \frac{1}{2^{n}} \bar{u}_{n}(x)
\end{gathered}
$$

is a Lipschitz continuous Lyapunov function for $\phi$ such that $\mathcal{N}(u)=\mathcal{S C R}(\phi)$.

Proof. The Lipschitz continuity of $u$ is a direct consequence of property $(i)$ of Lemma 4.2 . Indeed, for any $x, y \in X$, we have

$$
u(y)-u(x)=\sum_{n \in \mathbb{N}} \frac{1}{2^{n}}\left(\bar{u}_{n}(y)-\bar{u}_{n}(x)\right) \leq 4 d(x, y)
$$

and we conclude by exchanging the role of $x, y$.

Moreover, from property (ii) of Lemma 4.2 , it holds

$$
u\left(\phi_{t}(x)\right)-u(x)=\sum_{n \in \mathbb{N}} \frac{1}{2^{n}}\left(\bar{u}_{n}\left(\phi_{t}(x)\right)-\bar{u}_{n}(x)\right) \leq 0 \quad \forall x \in X \text { and } t \geq 0 .
$$

This means that $u$ is a Lyapunov function for $\phi$.

Finally, let $x \in X \backslash \mathcal{S C R}(\phi)$ and $t>0$. We first notice that the strong chain recurrent set for $\phi$-see $\left(5-\right.$ - can be equivalently expressed as $\mathcal{S C R}(\phi)=\bigcap_{n \in \mathbb{N}} \mathcal{A}_{\frac{n}{2}}$. As a consequence, if $x \in X \backslash \mathcal{S C R}(\phi)=X \backslash\left(\bigcap_{n \in \mathbb{N}} \mathcal{A}_{\frac{n}{2}}\right)=\bigcup_{n \in \mathbb{N}}\left(X \backslash \mathcal{A}_{\frac{n}{2}}\right)$, then there exists $\bar{n} \in \mathbb{N}$ such that $x \in X \backslash \mathcal{A}_{\overline{\frac{n}{2}}}$. By property (iii) of Lemma 4.2 , we then have

$$
\bar{u}_{\bar{n}}\left(\phi_{t}(x)\right)-\bar{u}_{\bar{n}}(x)<0
$$

and therefore

$$
u\left(\phi_{t}(x)\right)-u(x)=\sum_{\substack{n \in \mathbb{N} \\ n \neq \bar{n}}} \frac{1}{2^{n}}\left(\bar{u}_{n}\left(\phi_{t}(x)\right)-\bar{u}_{n}(x)\right)+\frac{1}{2^{\bar{n}}}\left(\bar{u}_{\bar{n}}\left(\phi_{t}(x)\right)-\bar{u}_{\bar{n}}(x)\right)<0 .
$$

This proves that $u$ is strict outside $\mathcal{S C R}(\phi)$, that is $\mathcal{N}(u) \subseteq \mathcal{S C R}(\phi)$. However, by Proposition 1.6 in [1, the neutral set of every Lipschitz continuous Lyapunov function for $\phi$ contains the strong chain recurrent set of $\phi$, so $\mathcal{S C R}(\phi) \subseteq \mathcal{N}(u)$. We then conclude that $\mathcal{N}(u)=\mathcal{S C R}(\phi)$.

The above theorem implies the following characterization of the strong chain recurrent set in terms of Lipschitz continuous Lyapunov functions. 
Corollary 4.1. Let $\phi: X \times \mathbb{R} \rightarrow X$ be a continuous flow on a compact metric space $(X, d)$, uniformly Lipschitz continuous on the compact subsets of $[0,+\infty)$. Then,

$$
\mathcal{S C R}(\phi)=\bigcap_{f \in \mathcal{L}(\phi)} \mathcal{N}(f)
$$

where $\mathcal{L}(\phi)$ denotes the set of all Lipschitz continuous Lyapunov functions for $\phi$.

Proof. By Proposition 1.6 in [1], the neutral set of every Lipschitz continuous Lyapunov function for $\phi$ contains the strong chain recurrent set of $\phi$, that is

$$
\mathcal{S C R}(\phi) \subseteq \bigcap_{f \in \mathcal{L}(\phi)} \mathcal{N}(f)
$$

Since -by Theorem 4.1 the function $u$ defined in 16 is such that $\mathcal{S C R}(\phi)=\mathcal{N}(u)$, the thesis immediately follows.

We stress that the corollary above has been already proved in [1][Theorem 2.2]. However, in [1] the inclusion $\supseteq$ is obtained by an accurate adaptation of Fathi and Pageault's techniques. While, in our proof, it is an immediate consequence of the existence of the Lyapunov function (16).

Let now $(M, g)$ be a $C^{\infty}$ closed connected Riemannian manifold and $V: M \rightarrow T M$ be a $C^{k}$ vector field, $k \geq 2$, inducing the flow $\phi: \mathbb{R} \times M \rightarrow M$. Denote by $\|v\|_{x}$ the norm of an element $v \in T_{x} M$ relatively to the metric $g$ and introduce the Mañé Hamiltonian

$$
H_{V}: T^{*} M \rightarrow \mathbb{R}, \quad H_{V}(x, p)=\frac{1}{2}\|p\|_{x}^{2}+p(V(x)) .
$$

We indicate by $\mathcal{A}_{V}$ the projected Aubry set associated to $H_{V}$. Since the constant functions are solutions of the Hamilton-Jacobi equation $H_{V}\left(x, d_{x} v\right)=0$, the Mañé critical level is $c\left(H_{V}\right)=0$. From one hand, it holds that $\mathcal{A}_{V} \subseteq \mathcal{C} \mathcal{R}(\phi)$, see e.g. [14][Section I, Theorem V], [16][Introduction, Section 3, Proposition 3.2] and [5] [Corollary 2]. From the other hand, we know that there exists a $C^{1,1}$ critical sub-solution $u: M \rightarrow \mathbb{R}$ of $H_{V}\left(x, d_{x} v\right)=0$ which is strict outside $\mathcal{A}_{V}$, see [4][Lemma 7]. In particular, $d_{x} u(V(x)) \leq 0$ and therefore $u$ is a $C^{1,1}$ Lyapunov function for $\phi$. Since $u$ is strict outside $\mathcal{A}_{V}$, it holds $\mathcal{N}(u) \subseteq \mathcal{A}_{V}$. Furthermore, recall that for any $x \in \mathcal{A}_{V}$ and any sub-solution $v$

$$
\frac{1}{2}\left\|d_{x} v\right\|_{x}^{2}+d_{x} v(V(x)) \leq 0,
$$

$d_{x} v$ does not depend on $v$ (see [17][Proposition 5.1.23]). Consequently, since the constant functions are (sub-)solutions of $H\left(x, d_{x} v\right)=0$, we have $d_{x} v=0$ on $\mathcal{A}_{V}$. In particular, this implies $d_{x} u(V(x))=0$ for all $x \in \mathcal{A}_{V}$ or equivalently $\mathcal{A}_{V} \subseteq \mathcal{N}(u)$. Summarizing, $u$ is a $\mathcal{C}^{1,1}$ Lyapunov function for $\phi$ with $\mathcal{N}(u)=\mathcal{A}_{V}$. Therefore, by the previous corollary, $\operatorname{SCR}(\phi) \subseteq \mathcal{A}_{V}$. From both inclusions, we conclude that in general

$$
\operatorname{SCR}(\phi) \subseteq \mathcal{A}_{V} \subseteq \mathcal{C R}(\phi)
$$

Corollary 4.2. If $\mathcal{S C R}(\phi)=\mathcal{C R}(\phi)$ then there exists a $\mathcal{C}^{1,1}$ Lyapunov function such that $\mathcal{N}(u)=$ $\operatorname{SCR}(\phi)$.

Proof. If $\mathcal{S C R}(\phi)=\mathcal{C R}(\phi)$ then necessarily $\mathcal{S C R}(\phi)=\mathcal{A}_{V}=\mathcal{C} \mathcal{R}(\phi)$ and the $C^{1,1}$ critical subsolution $u: M \rightarrow \mathbb{R}$ of $H_{V}\left(x, d_{x} u\right)=0$ which is strict outside $\mathcal{A}_{V}$ gives the desired Lyapunov function with $\mathcal{N}(u)=\mathcal{S C R}(\phi)$.

Next basic Example 4.1 shows two different smooth flows on $\mathbb{T}^{1}=\mathbb{R} / \mathbb{Z}$, having the following properties: 
(i) For the first flow, the hypothesis of Corollary 4.2 holds and therefore it exists a differentiable Lyapunov function which is strict outside the strong chain recurrent set.

(ii) For the second flow, we have that $\operatorname{SCR}(\phi) \varsubsetneqq \mathcal{A}_{V}=\mathbb{T}^{1}$. In such a case, even if every subsolution given by weak KAM theory provides a first integral for $\phi$, the dynamical system admits a differentiable Lyapunov function strict outside $\operatorname{SCR}(\phi)$.

Example 4.1. On the circle $\mathbb{T}^{1}=\mathbb{R} / \mathbb{Z}$ equipped with the standard quotient metric, consider the dynamical systems of Figures 1 and 2 , where the bold line and the arrows denote respectively fixed points and the direction of the flow $\phi$.

Relatively to the dynamical system of Figure 1, the hypothesis of Corollary 4.2 is satisfied since $\mathcal{S C R}(\phi)=\mathcal{C R}(\phi)=F i x(\phi)$ and it clearly exists a $C^{1,1}$ (also $C^{\infty}$ ) Lyapunov function whose neutral set is the strong chain recurrent set.

Otherwise, in the case of the dynamical system of Figure $2, \mathcal{S C R}(\phi)=F i x(\phi)$ and $\mathcal{C R}(\phi)=\mathbb{T}^{1}$. Moreover -see point $(i)$ of Theorem 4.8 and point $(i)$ of Lemma 4.14 in [11]- the projected Aubry set associated to the corresponding Mañé Hamiltonian coincides with $\mathcal{C} \mathcal{R}(\phi)=\mathbb{T}^{1}$. Consequently, every sub-solutions given by weak KAM theory is a first integral but-according to Theorem 4.1it clearly exists a Lipschitz continuous (even smooth) Lyapunov function strict outside Fix $(\phi)$.

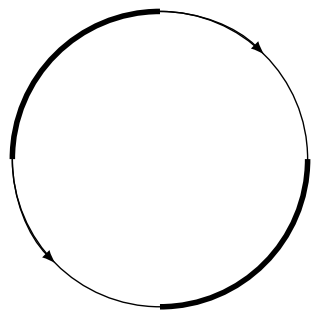

Figure 1: First dynamical system of Example 4.1 .

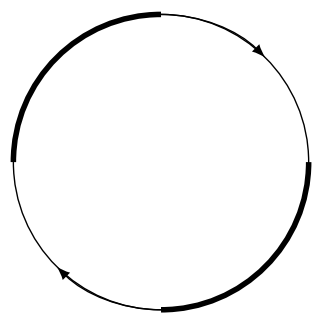

Figure 2: Second dynamical system of Example 4.1 .

Another case where sub-solutions of weak KAM theory provide first integrals while the Lyapunov function of Theorem 4.1 is not a first integral, is discussed in the next

Example 4.2. On $\mathbb{T}^{2}=\mathbb{R}^{2} / \mathbb{Z}^{2}$ endowed with the standard quotient metric, consider the flow $\phi$ associated to the vector field (see Figure 3):

$$
V(x, y)=(f(x), 1)
$$

where

$$
f(x)= \begin{cases}\cos (4 \pi x)+1 & x \in\left[0, \frac{1}{4}\right] \cup\left[\frac{3}{4}, 1\right) \\ 0 & x \in\left[\frac{1}{4}, \frac{3}{4}\right]\end{cases}
$$

In such a case, the strong chain recurrent set coincides with the set of periodic points, that is

$$
\operatorname{SCR}(\phi)=\left[\frac{1}{4}, \frac{3}{4}\right] \times \mathbb{T}^{1}
$$

and $\mathcal{C R}(\phi)=\mathbb{T}^{2}$. Moreover -by points $(i)$ of Theorem 4.8 and $(i)$ of Lemma 4.14 in [11] againthe projected Aubry set associated to the Mañé Hamiltonian

$$
\frac{1}{2}\left(p_{1}^{2}+p_{2}^{2}\right)+f(x) p_{1}+p_{2}
$$

is $\mathcal{A}_{V}=\mathcal{C} \mathcal{R}(\phi)=\mathbb{T}^{2}$. Consequently, every sub-solutions given by weak KAM theory is a first integral for $\phi$ while function (16) of Theorem 4.1 gives a Lyapunov function for $\phi$ which is strict outside $\left[\frac{1}{4}, \frac{3}{4}\right] \times \mathbb{T}^{1}$. 


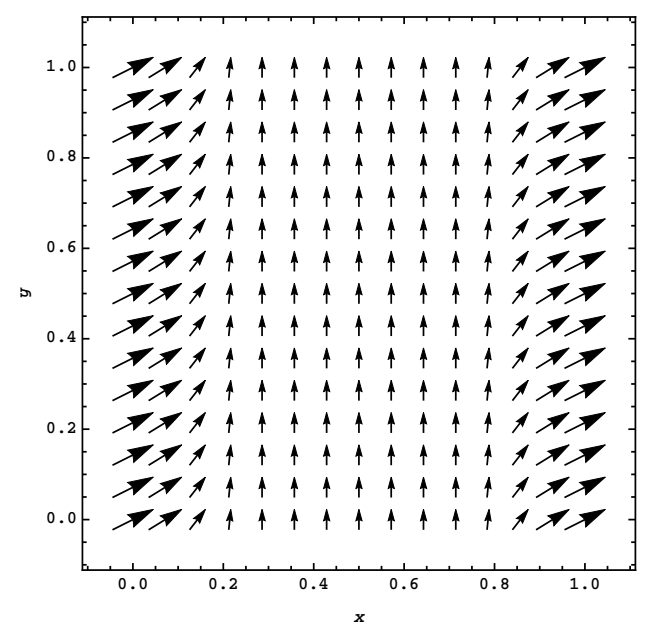

Figure 3: The vector field 19 .

We finally remark that the converse implication of Corollary 4.2 does not in general hold true. For example, consider the dynamical system of Figure 2, In such a case, it is easy to construct a smooth Lyapunov function which is strict outside $\mathcal{S C R}(\phi)=F i x(\phi)$. However, $\mathcal{S C R}(\phi) \varsubsetneqq \mathcal{C R}(\phi)=\mathbb{T}^{1}$. A necessary and sufficient condition for the strong chain recurrent and chain recurrent sets to be equal is proved in Proposition 5.3 of the next section.

\section{Strong chain transitivity and Lipschitz Lyapunov functions}

In this section, $\phi: X \times \mathbb{R} \rightarrow X$ is a continuous flow on a compact metric space $(X, d)$.

We first introduce $L_{T}$-dominated and $L$-dominated functions and then we explain their relation with Lipschitz continuous Lyapunov functions and the strong chain recurrent set. Next definitions and results are the continuous-time versions of the ones appearing in [16][Chapter 2, Section 4] and [12][Section 2.2] for homeomorphisms.

Definition 5.1. (Dominated function)

(i) A function $f: X \rightarrow \mathbb{R}$ is said to be $L_{T}$-dominated if there exists $K_{T}>0$ such that

$$
f(y)-f(x) \leq K_{T} L_{T}(x, y) \quad \forall x, y \in X .
$$

(ii) A function $f: X \rightarrow \mathbb{R}$ is said to be $L$-dominated if it is $L_{T}$-dominated for any $T>0$.

The proof of the next proposition is an easy adaptation for flows of the proof of Lemma 4.3 in [16].

Proposition 5.1. A Lipschitz continuous function $f: X \rightarrow \mathbb{R}$ is $L_{T}$-dominated if and only if it is definitively a Lyapunov function for $\phi$, that is

$$
f\left(\phi_{t}(x)\right) \leq f(x) \quad \text { for any } x \in X \text { and } t \geq T .
$$

Proof. From one hand, let $f: X \rightarrow \mathbb{R}$ be a $L_{T}$-dominated, Lipschitz continuous function for $\phi$. Thanks to point (ii) of Proposition 2.1, for any fixed $x \in X$ and $t \geq T$ it holds

$$
f\left(\phi_{t}(x)\right)-f(x) \leq K_{T} L_{T}\left(x, \phi_{t}(x)\right)=0,
$$

that is $f$ is a definitively Lyapunov function.

On the other hand, let $f: X \rightarrow \mathbb{R}$ be a $K$-Lipschitz continuous, definitively Lyapunov function for $\phi$. Fixed $x, y \in X$, let $C=\left(x_{i}, t_{i}\right)_{i=1, \ldots, n} \in C_{T}(x, y)$ be a chain from $x$ to $y$. Then

$$
f\left(x_{i+1}\right)-f\left(x_{i}\right) \leq f\left(x_{i+1}\right)-f\left(\phi_{t_{i}}\left(x_{i}\right)\right) \leq K d\left(\phi_{t_{i}}\left(x_{i}\right), x_{i+1}\right) \quad \forall i=1, \ldots, n .
$$


By adding all the inequalities, we conclude that

$$
f(y)-f(x)=\sum_{i=1}^{n}\left(f\left(x_{i+1}\right)-f\left(x_{i}\right)\right) \leq K \sum_{i=1}^{n} d\left(\phi_{t_{i}}\left(x_{i}\right), x_{i+1}\right)=K l_{T}(C) .
$$

More precisely, by considering the infimum over all possible chains in $C_{T}(x, y)$ we have

$$
f(y)-f(x) \leq K L_{T}(x, y) \quad \forall x, y \in X
$$

where $K$ is any Lipschitz constant for $f$.

Corollary 5.1. A Lipschitz continuous function $f: X \rightarrow \mathbb{R}$ is L-dominated if and only if it is a Lyapunov function for $\phi$.

In the sequel, we denote by $\mathcal{L}_{T}^{1}(\phi)$ the set of 1-Lipschitz continuous functions $f: X \rightarrow \mathbb{R}$ such that $f\left(\phi_{t}(x)\right) \leq f(x)$ for any $x \in X$ and $t \geq T$. The next lemma is the continuous-time version of Proposition 4.6 in [16].

Lemma 5.1. For any $x \in \mathcal{S C R}(\phi)$ and $y \in X$, we have

$$
L_{T}(x, y)=\sup _{f \in \mathcal{L}_{T}^{1}(\phi)} f(y)-f(x) .
$$

Proof. By the previous Proposition 5.1, if $f \in \mathcal{L}_{T}^{1}(\phi)$ then $f$ is $L_{T}$-dominated; moreover, see inequality (20), it results that $K_{T}=1$. This means that

$$
f(w)-f(z) \leq L_{T}(z, w) \quad \forall z, w \in X
$$

and consequently

$$
L_{T}(z, w) \geq \sup _{f \in \mathcal{L}_{T}^{1}(\phi)}(f(w)-f(z)) \quad \forall z, w \in X .
$$

In order to prove the other inequality, let us fix $x \in \mathcal{S C R}(\phi)$ and define $f_{x}(\cdot):=L_{T}(x, \cdot)$. We notice that the function $f_{x}(\cdot) \in \mathcal{L}_{T}^{1}(\phi)$. Indeed:

$$
f_{x}(z)-f_{x}(y)=L_{T}(x, z)-L_{T}(x, y) \leq d(y, z) \quad \forall y, z \in X
$$

and

$$
f_{x}\left(\phi_{t}(y)\right)-f_{x}(y)=L_{T}\left(x, \phi_{t}(y)\right)-L_{T}(x, y) \leq L_{T}\left(y, \phi_{t}(y)\right)=0
$$

for any $y \in X$ and $t \geq T$. Moreover, it holds that

$$
L_{T}(x, y)=L_{T}(x, y)-L_{T}(x, x)=f_{x}(y)-f_{x}(x)
$$

because $L_{T}(x, x)=0$. As a consequence,

$$
L_{T}(x, y) \leq \sup _{f \in \mathcal{L}_{T}^{1}(\phi)}(f(y)-f(x)) \quad \forall x \in \mathcal{S C R}(\phi) \text { and } y \in X .
$$

Previous Lemma 5.1 leads to prove the following results for the set $\mathcal{L}(\phi)$ of all Lipschitz continuous Lyapunov functions for $\phi$. The corresponding version for homeomorphisms is given by Corollary 4.7 and Theorem 4.8. of Chapter 2 in [16].

Proposition 5.2. Any $f \in \mathcal{L}(\phi)$ is constant on every strong chain transitive component of $\mathcal{S C R}(\phi)$. Moreover, if $x, y \in \mathcal{S C R}(\phi)$ belong to different strong chain transitive components of $\operatorname{SCR}(\phi)$, then there exists a function $f \in \mathcal{L}(\phi)$ such that $f(x) \neq f(y)$. 
Proof. Given $f \in \mathcal{L}(\phi)$ with Lipschitz constant $K_{f}>0$, it clearly holds that $\frac{f}{K_{f}} \in \mathcal{L}_{T}^{1}(\phi)$ for any $T>0$. Let now $x, y \in \mathcal{S C R}(\phi)$ be in the same strong chain transitive component of $\operatorname{SCR}(\phi)$, that is $L_{T}(x, y)=0=L_{T}(y, x)$ for all $T>0$. By equality (21), one has

$$
0=L_{T}(x, y)=\sup _{g \in \mathcal{L}_{T}^{1}(\phi)} g(y)-g(x) \geq \frac{f(y)}{K_{f}}-\frac{f(x)}{K_{f}}
$$

and

$$
0=L_{T}(y, x)=\sup _{g \in \mathcal{L}_{T}^{1}(\phi)} g(x)-g(y) \geq \frac{f(x)}{K_{f}}-\frac{f(y)}{K_{f}}
$$

so that $f(y)=f(x)$.

Conversely, let $x, y \in \mathcal{S C R}(\phi)$ be in different strong chain transitive components of $\mathcal{S C R}(\phi)$. Exchanging the role of $x, y$ if needed, this means that there exists a time $T>0$ such that $L_{T}(x, y)>0$. By using equality 21 again, we conclude that there exists a function $f \in \mathcal{L}_{T}^{1}(\phi)$ such that $f(y) \neq f(x)$. Let us now define

$$
\tilde{f}(z):=\max _{s \in[0, T]} f\left(\phi_{s}(z)\right)
$$

which is in $\mathcal{L}(\phi)$. Moreover, there exist $\bar{s}=\bar{s}(x) \in[0, T]$ and $\hat{s}=\hat{s}(y) \in[0, T]$ such that

$$
\tilde{f}(x)=f\left(\phi_{\bar{s}}(x)\right) \quad \text { and } \quad \tilde{f}(y)=f\left(\phi_{\hat{s}}(y)\right) .
$$

Since now -by Proposition 2.2 if $z \in \mathcal{S C R}(\phi)$ and $s \in \mathbb{R}$ then $z$ and $\phi_{s}(z)$ belong to the same strong chain transitive component of $\mathcal{S C R}(\phi)$, we conclude that

$$
\tilde{f}(x)=f\left(\phi_{\bar{s}}(x)\right)=f(x) \quad \text { and } \quad \tilde{f}(y)=f\left(\phi_{\hat{s}}(y)\right)=f(y) .
$$

As a consequence, we have proved that $\tilde{f} \in \mathcal{L}(\phi)$ is such that $\tilde{f}(x) \neq \tilde{f}(y)$.

The proof of the next theorem is essentially the same of Theorem 4.8 in Chapter 2 in [16] and it is omitted. We notice that Fathi and Pageault's statement is formulated by using the so-called $d$-Mather classes, corresponding exactly to the strong chain transitive components of the strong chain recurrent set for a given homeomorphism.

Theorem 5.1. Let $\phi: X \times \mathbb{R} \rightarrow X$ be a continuous flow on a compact metric space $(X, d)$. $\operatorname{SCR}(\phi)$ has a unique strong chain transitive component if and only if the only Lipschitz continuous Lyapunov functions for $\phi$ are the constants.

In the fundamental theorem of dynamical systems recalled in the introduction (see [7][Chapter II]), Conley made explicit a so-called complete Lyapunov function for $\phi$, whose properties are listed below.

Definition 5.2. (Complete Lyapunov function)

Let $\phi: X \times \mathbb{R} \rightarrow X$ be a continuous flow on a compact metric space $(X, d)$. A continuous Lyapunov function $f: X \rightarrow \mathbb{R}$ for $\phi$ is called complete if:

(i) $\mathcal{N}(f)=\mathcal{C R}(\phi)$.

(ii) If $x, y \in \mathcal{C R}(\phi)$, then $f(x)=f(y)$ if and only if $x$ and $y$ belong to the same chain transitive component of $\mathcal{C} \mathcal{R}(\phi)$.

(iii) $f(\mathcal{C R}(\phi))$ is a compact nowhere dense subset of $\mathbb{R}$.

We notice that the function given by Theorem 4.1 cannot be in general assumed to be complete with respect to $\mathcal{S C R}(\phi)$. For example, look at the dynamical system of Figure 2, In such a case, every fixed point is a strong chain transitive component of $\mathcal{S C R}(\phi)=$ Fix $(\phi)$ but it is not possible to construct a continuous Lyapunov function such that properties: 
$(i)^{\prime} \mathcal{N}(f)=\mathcal{S C R}(\phi)$

and

$(\text { ii })^{\prime}$ If $x, y \in \mathcal{S C R}(\phi)$, then $f(x)=f(y)$ if and only if $x$ and $y$ belong to the same strong chain transitive component of $\mathcal{S C R}(\phi)$

simultaneously hold. In particular, if we change the setting from chain recurrence to strong chain recurrence, the next notion of pseudo-complete Lyapunov function is crucial. This definition was first introduced (at least at our knowledge) by Katsuya Yokoi for homeomorphisms (see [19][Definition 5.1] and also [18][Section 6]).

Definition 5.3. (Pseudo-complete Lyapunov function)

Let $\phi: X \times \mathbb{R} \rightarrow X$ be a continuous flow on a compact metric space $(X, d)$. A continuous Lyapunov function $f: X \rightarrow \mathbb{R}$ for $\phi$ is called pseudo-complete if $\mathcal{N}(f)=\mathcal{S C R}(\phi)$ and $f$ is constant on every strong chain transitive component of $\mathcal{S C R}(\phi)$.

Indeed, from Theorem 4.1 and the first part of Proposition 5.2, we immediately deduce the next

Corollary 5.2. Let $\phi: X \times \mathbb{R} \rightarrow X$ be a continuous flow on a compact metric space $(X, d)$, uniformly Lipschitz continuous on compact subsets of $[0,+\infty)$. Then there exists a pseudocomplete Lyapunov function for $\phi$.

We finally remark that the notion of pseudo-complete Lyapunov function is useful to discuss the condition for the strong chain recurrent and chain recurrent sets to be equal (see [19][Theorem 5.3] for the case of a homeomorphism).

Proposition 5.3. Let $\phi: X \times \mathbb{R} \rightarrow X$ be a continuous flow on a compact metric space $(X, d)$. Then, $\mathcal{S C R}(\phi)=\mathcal{C} \mathcal{R}(\phi)$ if and only if there exists a pseudo-complete Lyapunov function $u: X \rightarrow$ $\mathbb{R}$ for $\phi$ such that $u(\mathcal{S C R}(\phi))$ is totally disconnected.

Proof. Let $\mathcal{S C R}(\phi)=\mathcal{C R}(\phi)$. By Conley's fundamental theorem (see [7][Chapter II, Section $6.4]$ ), there exists a complete Lyapunov function $u$ for $\phi$. Clearly, $u$ is also a pseudo-complete Lyapunov function for $\phi$ such that $u(\mathcal{S C R}(\phi))=u(\mathcal{C R}(\phi))$ is totally disconnected.

Conversely, let $u: X \rightarrow \mathbb{R}$ be a pseudo-complete Lyapunov function for $\phi$ such that $u(\mathcal{S C R}(\phi))$ is totally disconnected. Without loss of generality, we suppose that $u(x) \geq 0$ for any $x \in X$. In order to show that $\mathcal{C R}(\phi) \subseteq \mathcal{S C R}(\phi)$, we remind that the next conditions are equivalent:

(i) $x \notin \mathcal{C R}(\phi)$.

(ii) There exists an attractor $K$ such that $x \notin K$ but $\omega(x) \subseteq K$.

See [7][Chapter II, Section 6.2, Page 37] for this equivalence. Let $x \notin \mathcal{S C R}(\phi)$ and $\bar{t}>0$ such that $u\left(\phi_{\bar{t}}(x)\right)<u(x)$. Since $u(\mathcal{S C R}(\phi))$ is totally disconnected, there exists $r_{0}>0$ such that $u\left(\phi_{\bar{t}}(x)\right)<r_{0}<u(x)$ and $r_{0} \notin u(\mathcal{S C R}(\phi))$. We define $U:=\left\{y \in X: u(y) \in\left[0, r_{0}\right)\right\}$. Since $u$ is a Lyapunov function for $\phi$, we have that $K:=\omega(U) \subseteq U$, which means that $K$ is an attractor. Moreover, $x \notin K$ but, since $\phi_{\bar{t}}(x) \in U, \omega(x) \subseteq K$. From the equivalence of points $(i)$ and $(i i)$ recalled above, we conclude that $x \notin \mathcal{C} \mathcal{R}(\phi)$. This prove that $\mathcal{C} \mathcal{R}(\phi) \subseteq \mathcal{S C R}(\phi)$ and therefore $\mathcal{C R}(\phi)=\mathcal{S C R}(\phi)$.

\section{References}

[1] A. Abbondandolo, O. Bernardi, And F. Cardin, Chain recurrence, chain transitivity, Lyapunov functions and rigidity of Lagrangian submanifolds of optical hypersurfaces, Journal of Dynamics and Differential Equations, (on line first 2016). 
[2] E. Akin, The general topology of dynamical systems, vol. 1 of Graduate Studies in Mathematics, American Mathematical Society, Providence, RI, 1993.

[3] E. Akin and J. Wiseman, Chain recurrence for general spaces, arXiv:1707.09601 (2017).

[4] P. Bernard, Existence of $\mathcal{C}^{1,1}$ critical sub-solutions of the Hamilton-Jacobi equation on compact manifolds, Annales Scientifiques de l'École Normale Supérieure. Quatriéme Série, 40 (2007), pp. 445-452.

[5] — On the Conley decomposition of Mather sets, Revista Matemática Iberoamericana, 26 (2010), pp. 115-132.

[6] O. Bernardi And A. Florio, A Conley-type decomposition of the strong chain recurrent set, Ergodic Theory and Dynamical Systems, (to appear).

[7] C. Conley, Isolated invariant sets and the Morse index, vol. 38 of CBMS Regional Conference Series in Mathematics, Regional Conference Series in Mathematics, American Mathematical Society, Providence, R.I., 1978.

[8] — The gradient structure of a flow. I, Ergodic Theory and Dynamical Systems, 8* (1988), pp. $11-26,9$.

[9] R. Easton, Chain transitivity and the domain of influence of an invariant set, in The structure of attractors in dynamical systems (Proc. Conf., North Dakota State Univ., Fargo, N.D.), vol. 668 of Lecture Notes in Math., Springer, Berlin, 1978, pp. 95-102.

[10] A. Fathi, The Weak KAM Theorem in Lagrangian Dynamics, Cambridge University Press, (to appear).

[11] A. Fathi, A. Figalli, And L. Rifford, On the Hausdorff dimension of the Mather quotient, Communications on Pure and Applied Mathematics, 62 (2009), pp. 445-500.

[12] A. Fathi And P. Pageault, Aubry-Mather theory for homeomorphisms, Ergodic Theory and Dynamical Systems, 35 (2015), pp. 1187-1207.

[13] J. Franks, Notes on Chain Recurrence and Lyapunov Functions, arXiv:1704.07264 (2017).

[14] R. MAÑé, Lagrangian flows: the dynamics of globally minimizing orbits, Boletim da Sociedade Brasileira de Matemática. Nova Série, 28 (1997), pp. 141-153.

[15] D. Norton, The fundamental theorem of dynamical systems, Commentationes Mathematicae Universitatis Carolinae, 36 (1995), pp. 585-597.

[16] P. Pageault, Lyapunov functions: a weak KAM approach, PhD thesis, Ecole normale supérieure de Lyon-ENS LYON, Nov. 2011.

[17] A. Sorrentino, Lecture notes on Mather's theory for Lagrangian systems, Publicaciones Matemáticas del Uruguay, 16 (2016), pp. 169-192.

[18] J. Wiseman, The generalized recurrent set and strong chain recurrence, Ergodic Theory and Dynamical Systems, (on line first 2016).

[19] K. YokoI, On strong chain recurrence for maps, Annales Polonici Mathematici, 114 (2015), pp. $165-177$.

[20] Z. Zheng, Attractor, chain recurrent set and limit set of flow, Science in China. Series A. Mathematics, 43 (2000), pp. 244-251. 